\title{
Heart Rate Variability Analysis During Weaning from Mechanical Ventilation: Models for Prediction of the Weaning Trial Outcome
}

\author{
Vessela Krasteva $^{1}$, Mikhail Matveev ${ }^{1}$, Irena Jekova ${ }^{1}$, Georgi Georgiev ${ }^{2}$ \\ ${ }^{1}$ Institute of Biophysics and Biomedical Engineering, Bulgarian Academy of Sciences, Sofia, Bulgaria \\ ${ }^{2}$ Pirogov University Emergency Hospital, Sofia, Bulgaria
}

\begin{abstract}
This study estimates the activity of the autonomic cardiac control (ACC) in patients undergoing weaning from mechanical ventilation, aiming to predict the weaning outcome. ECG and AVEA ventilator data from 13 successfully and 14 unsuccessfully weaned patients were collected. Heart rate variability (HRV) profiles were estimated in non-sedated patients during 2 weaning phases: (1) PSV - pressure support ventilation at 12-25 $\mathrm{CmH}_{2} \mathrm{O}$; (2) SBT - spontaneous breathing trial at 8 $\mathrm{CmH}_{2} \mathrm{O}$. HRV indices in the time- and frequency-domain were analyzed on 5-min RR-interval episodes under stationary conditions during each weaning phase.

Our model for prediction of the successful weaning outcome considers 3 basic mechanisms for adequate ACC response on the weaning cardio-respiratory stress: (1) preserved ACC ability to maintain physiological adaptation; (2) modulated ACC activity by the breathing model; (3) mostly neurohumoral regulation of the blood circulation. The successful group model exhibits reduced total activity (TP, SDNN) with increased sympathetic (VLF/TP, LF/HF>1) and reduced vagal tone (HF/TP, RMSSD, pNN50), the latter correlated to the respiratory rate and tidal volume. Deviations from this HRV model are indicative for weaning failure detected with accuracy 92.6\% (PSV), 81.5\% (SBT), 96.3\% (SBT-PSV).
\end{abstract}

\section{Introduction}

Mechanical ventilation (MV) of critically ill patients is associated with many risks and complications that lead to high morbidity, mortality, longer intensive care unit stay, and higher treatment costs [1]. Identifying the patient's readiness at the earliest time possible for successful weaning from MV is the primary target for patients with command breathing [2]. Discontinuation from MV is the process for a gradual reduction in ventilator support, to allow patients the ability to assume increasing levels of work to breathe until sustain spontaneous breathing. This weaning process occupies an average of $40 \%$ of the total duration of mechanical ventilation, with failure in over one third of MV patients. The consolidated evidencebased clinical practice guidelines of the American Thoracic Society (ATS) and the American College of Chest Physicians (CHEST) point out the process of MV liberation as an investigation priority [1].

Weaning from MV has impact on the cardiovascular function by 3 physiological mechanisms [3-6]:

1) Changes in the oxygen transport (hypoxia), which may provoke circulatory disturbances with a terminal result of weaning failure or other fatal complications (acute myocardial infarction, heart failure and cardiogenic pulmonary edema, rhythm disorders).

2) Haemodynamic alterations as a result of changes in the intrathoracic pressure (ITP), which is positively dependent on the tidal volume and modulated by MV breathing cycles [6]. The associated changes in the cardiac output, ventricular preload and afterload may result in acute alterations of cardiac mechanics and myocardial ischemia, manifested as arrhythmias.

3) Effects on the autonomic nervous system (ANS) activity, which is influenced by humoral changes in the intrathoracic cardiovascular system due to ITP changes. Therefore, ANS tries to compensate for these humoral changes by two mechanisms - increasing the sympathetic tone and decreasing the parasympathetic tone that affects the heart rate.

Heart rate variability (HRV) is the physiological phenomenon of inter-beat interval variation due to the joint action of the sympathetic and parasympathetic parts of the autonomic cardiac control (ACC) [7]. A number of studies on HRV changes at different phases during MV discontinuation have concluded that ACC status provides essential information on the pathophysiological imbalances reflected in the success or failure of weaning [3-5,8-14]. In failure patients, reduced HRV and vagal tone withdrawal have been reported $[9,10,12]$. This study aims to derive models for prediction of the weaning outcome by HRV, breathing and metabolic features, and to better understand the ACC role during MV weaning. 


\section{Materials and methods}

\subsection{Study population}

Data from 27 patients undergoing weaning from MV (63\% men, age $58 \pm 17$ years, Simplified Acute Physiology Score SAPS II $=28.9 \pm 8.2$ ) were collected with AVEA ventilator system in the intensive care unit of the Pirogov University Emergency Hospital, Sofia. According to inclusion criteria, the enrolled patients did not have cardiac arrhythmias, neurological diseases, did not take pre-medication with cardiovascular drugs, and received CMV for at least 72 hours prior the study. The decision to start weaning and weaning outcome has been made by the primary care physician following a weaning protocol approved by the local Ethics Committee, in concord to general weaning and extubation criteria [15].

The study considered two weaning phases in nonsedated patients:

1) Pressure support ventilation (PSV): titration of the inspiratory pressure support level from 25 down to $12 \mathrm{cmH}_{2} \mathrm{O}$ during about $30 \mathrm{~min}$. All patients have successfully passed this phase.
2) Spontaneous breathing trial (SBT): PSV at $8 \mathrm{cmH}_{2} \mathrm{O}$. SBT was terminated if the patient exhibited signs of poor tolerance.

The weaning outcome was estimated by the ability of the patient to maintain the SBT challenge for at least 2 hours, being successful ( $\mathrm{S}=13$ patients) and failed $(\mathrm{F}=14$ patients).

\subsection{HRV analysis}

ECG signal (lead I, 500Hz) was continuously recorded. Time series of normal-to-normal RR-intervals (NN), deduced from adjacent normal sinus beats in stable artifact-free 5-min episodes under stationary conditions were extracted after the beginning of PSV and SBT. Standardized HRV indices [7] were calculated (Table 1):

- TIME-HRV from the time-domain NN-tachogram, $\mathrm{dNN}$-tachogram (NN-intervals first differences) and NNhistogram (using standard discrete scale resolution of the bin equal to $7.8 \mathrm{~ms}=1 / 128 \mathrm{~Hz}$ ).

- FREQ-HRV. Fourier Transform of NN interval time series resampled at $4 \mathrm{~Hz}$ was applied to derive the power spectrum density components of HRV.

Table 1. Mean \pm standard deviation of time-frequency domain HRV, ventilatory and hemodynamic indices for failure ( $\mathrm{F}=14$ patients) and successful group ( $\mathrm{S}=13$ patients) during PSV and SBT. *: $\mathrm{p}<0.05$ comparing F vs. $\mathrm{S}$ groups by T-test.

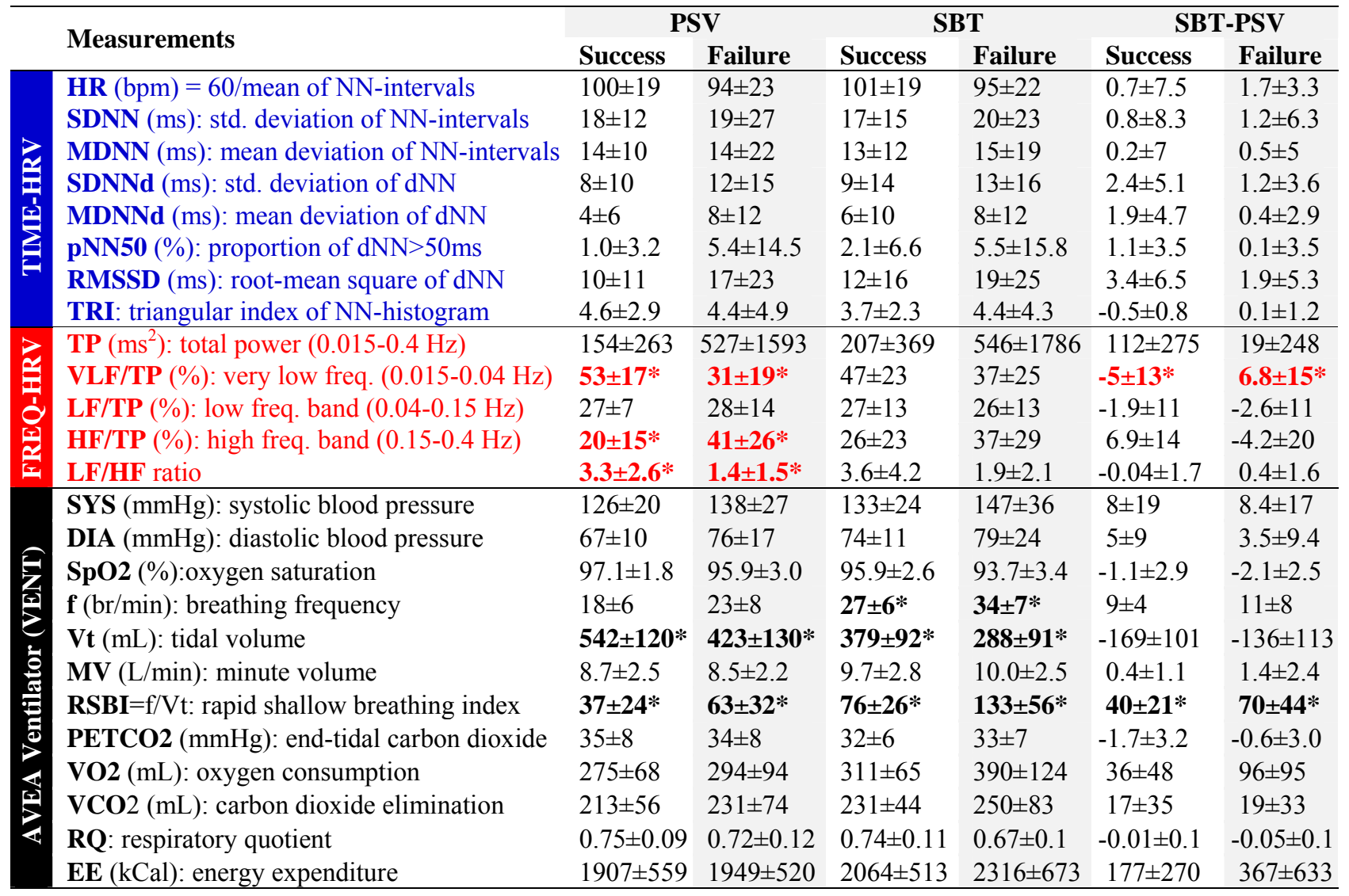




\subsection{Weaning outcome prediction model}

Both HRV indices and AVEA ventilator measurements (Table 1) were processed in Matlab (MathWorks, Inc.) by forward stepwise linear discriminant analysis (SDA) to derive models for prediction of the weaning outcome. Models using different input vectors were trained, aiming to select the most powerful and non-redundant predictors, measurable during PSV, SBT, and the difference (SBTPSV). The feature selection process was iterative, so that at each step, SDA was forced to include the predictor, which led to maximal classification accuracy:

$$
\text { Acuracy }=\frac{T P+T N}{T P+F N+T N+F P},
$$

where TP, FN are true positives and false negatives for detection of the weaning failure group ( $\mathrm{F}=14$ patients); $\mathrm{TN}, \mathrm{FP}$ are true negatives and false positives for detection of the weaning success group ( $\mathrm{S}=13$ patients).

Leave-one-out cross-validation was applied to derive the maximal classification accuracy achieved by SDA. Moreover, to ovoid overtraining on the small size database, SDA was limited to include up to 7 features or the training stopped while reaching a plateau in the accuracy step-up trend for $>2$ steps.

\section{Results and discussion}

The mean value dispersion (Table 1) of linked FREQHRV and TIME-HRV features provide reliable evidence for the cardiac autonomic balance (CAB) interpretation:

- Vagal activity is 1.6 to 5-fold higher in Failure patients, confirmed by the comparison (F vs. S) of:

- HF/TP: $41 \%$ vs. $20 \%$ (PSV), 37 vs. $26 \%$ (SBT)

- pNN50:5.4\% vs. $1 \%$ (PSV), $5.5 \%$ vs. $2.1 \%$ (SBT)

- RMSSD: 17 vs. 10 ms (PSV), 19 vs. 12 ms (SBT).

- Sympathetic activity is 1.3 to 2.4-fold higher in Successful patients, confirmed by comparison (S vs. F) of:

- VLF/TP: $53 \%$ vs. $31 \%$ (PSV), $47 \%$ vs. $37 \%$ (SBT)

- LF/HF ratio: 3.3 vs. 1.4 (PSV), 3.6 vs. 1.9 (SBT)

- LF/TP is indifferent to the weaning outcome (26$28 \%$ ), representing mixed sympathetic and vagal activity. LF is not appropriate as a marker of the sympathetic tone, as widely interpreted [8-10,13].

- Sympathovagal balance during PSV to SBT transition shows different $\mathrm{ACC}$ reaction:

- Failure patients become more stressed (VLF increases by $6.8 \%$, HF decreases by $4.2 \%$ ).

- Successful patients become more relaxed (VLF decreases by $5 \%$, HF increases by $6.9 \%$ ).
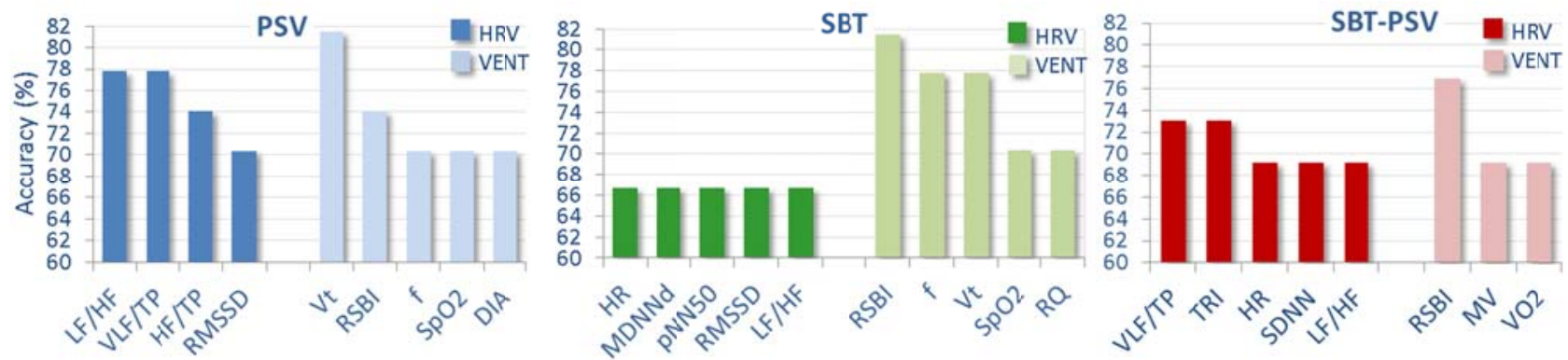

Figure 1. Top-ranked features (HRV, breathing, metabolic) in PSV, SBT, SBT-PSV for prediction of the weaning outcome.
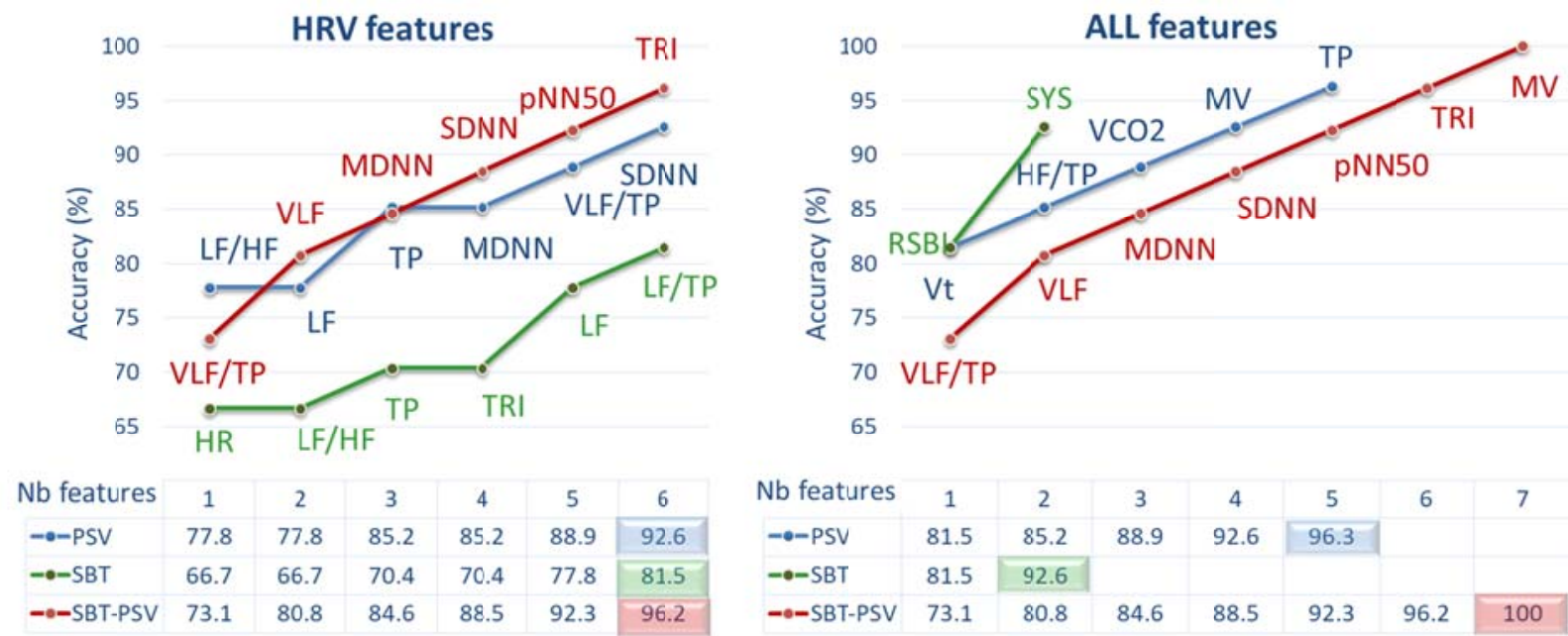

Figure 2. Performance of 6 SDA models for prediction of the weaning outcome, showing the iteratively selected features (only HRV - left graph, all features - right graph) in PSV, SBT, SBT-PSV that lead to maximization of accuracy. 


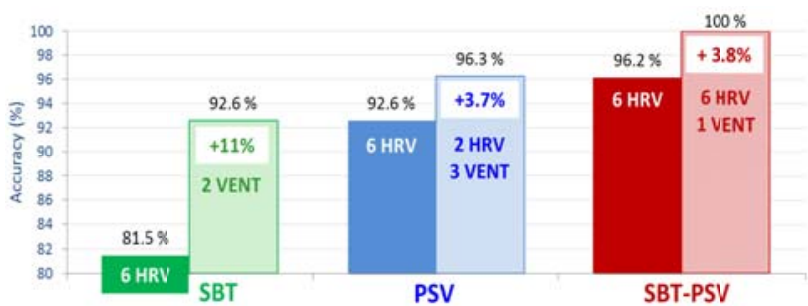

Figure 3. Final model for prediction of the weaning outcome: accuracy, number and type of included features.

The above analysis provides evidence that patients who succeed and failed during the weaning process have important differences in the ANS regulation:

1) Successful patients have prevalently sympathetic modulation, also confirmed in $[8,11]$. They maintain:

- Physiologic adaptation to the cardio-respiratory stress, immediately after the beginning of PSV.

- Intensive sympatho-adrenal stimulation to return the normal circulation (VLF $\uparrow$ )

- HRV modulation by the breathing pattern (HF $\downarrow$, $\mathrm{LF} / \mathrm{HF} \uparrow$ when $\mathrm{f} \uparrow, \mathrm{Vt} \downarrow$ ).

2) Failure patients keep high tension of the autonomic contour. They manifest:

- Increased vagal tone (HF $\uparrow, \mathrm{LF} / \mathrm{HF} \downarrow)$ and considerable HRV (TP $\uparrow, \mathrm{SDNN} \uparrow)$ during PSV, SBT.

- Slow physiologic adaptation:

o Later trend to increase of the sympathetic activity (transition PSV to SBT).

o This phenomenon was interpreted in some studies as "vagal tone withdrawal" $[9,10,12]$.

In this study, we stress the importance of the following HRV features to the prediction of the weaning outcome:

- VLF component, distinguishing the neurohumoral regulatory mechanisms during $\mathrm{MV}$ weaning [3]. The incomplete FREQ-HRV model $(\mathrm{TP}=\mathrm{LF}+\mathrm{HF})$ in many studies $[8-10,13]$ is deficient, also confirmed by the observed LF invariance to the weaning outcome.

- Both TIME-HRV and FREQ-HRV analyses provide the ground for reliable $\mathrm{CAB}$ activity interpretation by established strong correlations between pairs of timeand frequency-domain HRV indices. Study of only time-domain HRV [3] or highly variable frequencydomain HRV (mainly LF and HF) [9-11,13,14] can lead to interpretation bias, e.g. considering the effect of LF saturation at significant sympathetic ACC tone.

The prognostic value of HRV to the weaning outcome is significant, comparable to the breathing pattern features. The most powerful models are shown to be:

1) HRV-features (Figures 1-3):

o $\quad$ PSV $(\mathrm{LF} / \mathrm{HF}$ or $\mathrm{VLF} / \mathrm{TP})=78 \%$

o $\quad$ SBT-PSV (6 HRV features) $=96.2 \%$

2) Ventilator VENT-features (Figure 1):

o $\quad$ PSV $(\mathrm{Vt})$, SBT $(\mathrm{RSBI})=81 \%$

3) All features: HRV + VENT (Figures 2, 3):

o $\quad$ SBT-PSV $(6 \mathrm{HRV}+1 \mathrm{VENT})=100 \%$.

\section{References}

[1] Schmidt GA, Girard TD, Kress JP, et al. Liberation From Mechanical Ventilation in Critically Ill Adults: Executive Summary of an Official American College of Chest Physicians/ American Thoracic Society Clinical Practice Guideline. Chest 2017; 151:160-5.

[2] Haas CF, Loik PS. Ventilator discontinuation protocols. Respir Care 2012; 57:1649-62.

[3] Frazier SK, Moser DK, Schlanger R, Widener J, Pender L, Stone KS. Autonomic tone in medical intensive care patients receiving mechanical ventilation and during a CPAP weaning trial. Biol Res Nurs 2008; 9:301-10.

[4] Frazier SK, Stone KS, Moser D, Schlanger R, Carle C, Pender L. Hemodynamic changes during discontinuation of mechanical ventilation in medical intensive care unit patients. Am J Crit Care 2006; 15:580-93.

[5] Hammash MH. Cardiac rhythm during mechanical ventilation and weaning from ventilation. University of Kentucky Doctoral Dissertations 2010; 56.

[6] Lansdorp B, Hofhuizen C, Lavieren M, et al. Mechanical ventilation-induced intrathoracic pressure distribution and heart-lung interactions. Crit Care Med 2014; 42:1983-90.

[7] The Task Force of ESC and NASPE. Heart rate variability. Standards of measurement, physiological interpretation, and clinical use. Eur Heart J 1996; 17:354-81.

[8] Orini M, Giraldo B, Bailon R, et al. Time-frequency analysis of cardiac and respiratory parameters for the prediction of ventilator weaning. Conf Proc IEEE Eng Med Biol Soc 2008; 2008: 2793-6.

[9] Papaioannou V, Chouvarda I, Maglaveras N, Dragoumanis C, Pneumatikos I. Changes of heart and respiratory rate dynamics during weaning from mechanical ventilation: A study of physiologic complexity in surgical critically ill patients. J Crit Care 2011; 26:262-72.

[10] Shen HN, Lin LY, Chen KY, et al. Changes in heart rate variability during ventilator weaning. Chest 2003; 123:1222-8.

[11] Huang CT, Tsai YJ, Lin JW, Ruan SY, Wu HD, Yu CJ. Application of heart-rate variability in patients undergoing weaning from mechanical ventilation. Crit Care 2014, 18:R21.

[12] Guerra M, Igreja TP, de Carvalho TD, et al. Heart rate variability during weaning mechanical ventilation. J Cardiol Ther 2016; 3:519-23.

[13] Briceno FAC, Rojas CG, Sepúlveda DA, et al. Heart rate variability predicts success in weaning process. WCPT Congress 2015/Physiotherapy 2015; 101(S1): eS265.

[14] Arcentales A, Caminal P, Diaz I, Benito S, Giraldo BF. Classification of patients undergoing weaning from mechanical ventilation using the coherence between heart rate variability and respiratory flow signal. Physiol Meas 2015, 36: 1439-52.

[15] Stawicki SP. Mechanical ventilation: weaning and extubation. OPUS 12 Scientist 2007; 1:13-16.

Address for correspondence.

Vessela Krasteva

Institute of Biophysics and Biomedical Engineering

Acad. G. Bonchev str., bl.105, 1113, Sofia, Bulgaria vessika@biomed.bas.bg 